\title{
Comparison of Linear and Nonlinear MPC on Operator-In-the-Loop Overhead Cranes
}

\author{
Marco Giacomelli \\ Department of Mechanical and Industrial Engineering, University of Brescia \\ m.giacomelli009@unibs.it \\ Davide Colombo \\ Gefran Drives and Motion Srl, Gerenzano (VA), Italy \\ davide.colombo@gefran.com \\ Marco Faroni \\ Department of Mechanical and Industrial Engineering, University of Brescia \\ m.faroni003@unibs.it \\ Olivier Schmidt \\ Siemens Digital Industries Software, Lyon, France \\ olivier.schmidt@siemens.com \\ Luca Simoni \\ Department of Mechanical and Industrial Engineering, University of Brescia \\ luca.simoni@unibs.it \\ Antonio Visioli \\ Department of Mechanical and Industrial Engineering, University of Brescia \\ antonio.visioli@unibs.it
}

This is the pre-peer reviewed version of the following article: Comparison of Linear and Nonlinear MPC on Operator-In-the-Loop Overhead Cranes, which has been published in final form at DOI: 10.1109/ICCMA46720.2019.8988758.

This article may be used for non-commercial purposes in accordance with Journal terms and conditions for Self-Archiving.

\begin{abstract}
Model Predictive Control has been proved to enhance the control performance of overhead cranes. However, in OperatorIn-the-Loop (OIL) overhead cranes the trajectory of the payload strongly depends on the runtime decisions of the user and can not be predicted beforehand. Simple assumptions on the future references evolution have therefore to be made. In this paper we investigate the applicability of linear and nonlinear MPC strategies to the case of OIL overhead cranes, based on different assumptions on the future evolution of the length of the hoisting cable.
\end{abstract}

Index Terms

MPC, control, oscillations, crane, mechatronics

\section{INTRODUCTION}

Overhead cranes are the most widespread material handling systems in industry. Since they are underactuated underdamped systems, their control is not trivial. A poor control of an overhead crane can generate important oscillations of the payload during and after manoeuvres, increasing the positioning time and posing a safety hazard for the operators. A number of techniques have been proposed in the last fifty years for the control of these systems [1].

Among open-loop techniques, which aim to reduce the residual oscillations after the manoeuvre, input shaping is by far the most widespread [2]. Input-output inversion based techniques have also been proposed for both simple and double pendulum modelled overhead cranes [3], [4], [5]. While open-loop techniques are usually easy to implement on off-the-shelf industrial overhead cranes, as they do not require the presence of a sensor for the measure and the feedback of the system output or states, the decreasing cost of sensors have motivated an increasing focus on closed-loop approaches. The use of closed-loop techniques

This work has been developed within the European Union H2020 program ECSEL-2016-1 under grant agreement n. 737453 (I-MECH). 


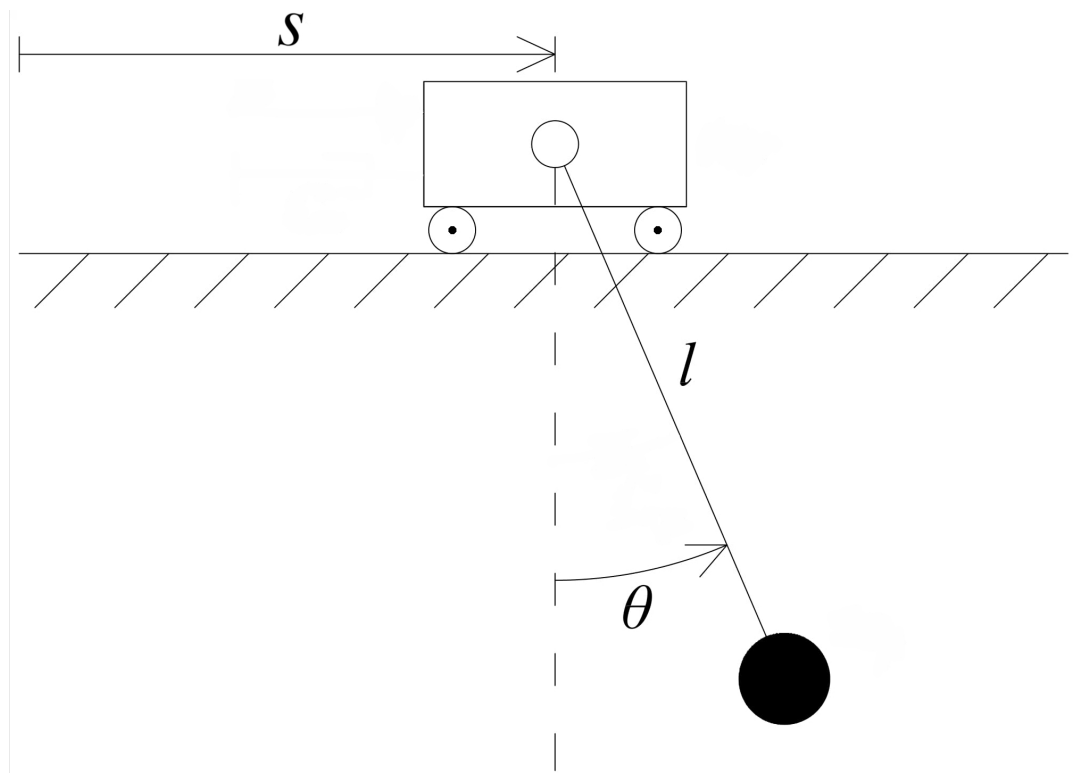

Fig. 1: Approximation of an overhead crane as a simple pendulum on a sliding cart.

brings, in general, an increased robustness and can lead to zero oscillation of the payload after the manoeuvre. Fuzzy logic closed-loop approaches have been proposed in [6], while adaptive control is used in order to compensate for varying parameters in [7]. On another side, the increment of standard industrial controllers computational capability has brought the focus on the application of Model Predictive Control (MPC) for the control of systems with relatively fast time constants such as overhead cranes and mechatronic systems in general. The MPC approach for the control of overhead cranes has been demonstrated to yield better performance than standard open-loop techniques in [8]. MPC algorithms have been used for position control of overhead cranes in [9], [10]. In [11], [12] MPC algorithms have been used to track offline computed trajectories, while in [13], [14] energy optimal MPC is applied for point-to-point motion. Other MPC techniques applied to the position control of overhead cranes can be found in [15], [16], [17].

The aforementioned MPC techniques focus on point-to-point motions or tracking of offline-computed trajectories. This approach is effective for completely automated systems, but it does not satisfy the requirements of cranes manoeuvered by an operator. As a matter of fact, in this case, the user does not operate in terms of point-to-point motions, but instead he/she gives a velocity reference to the system by pressing a button. Therefore, the control problem in manually operated cranes is completely different, in particular because it is not possible to know in advance the future velocity reference given each instant by the operator, while for the position control of autonomous overhead cranes the position reference is known in advance and does not change along the predictive horizon.

The control of Operator-In-the-Loop (OIL) overhead cranes has been addressed in [18]. Therein, the approach considers only the horizontal travel of the payload with constant cable length, limiting the point-to-point manoeuvres to be executed with separated and mutual-excluding sequences of horizontal travelling, hoisting and lowering, thereby increasing the total operation time.

In this paper, we investigate the applicability of different MPC techniques to OIL overhead cranes by explicitly taking into account the problem of controlling the crane during a manoeuvre which includes simultaneous payload horizontal travelling and hoisting. In particular, the focus is posed on the comparison between linear and nonlinear MPC approaches to the problem. While NMPC has been demonstrated to improve the performance on path-following tasks, its effectiveness in controlling cranes with non-predefined cable lengthening, such is the case of OIL cranes, has still to be investigated.

Moreover, the implementation of NMPC approaches on off-the-shelf industrial hardware is still non-trivial as it requires nonlinear quadratic solvers, while the implementation of linear MPC techniques is now the standard and usually leads decreased computational effort [19].

\section{MODELLING OF OVERHEAD CRANE SYSTEM}

When modelling an overhead crane, a common approximation is to consider it as a single pendulum on a sliding cart with the rope modelled as an inflexible rod, as shown in Figure 1, where $s$ is the position of the cart, $\theta$ is the angle between the vertical and the cable, and $l$ is the length of the cable.

A nonlinear model of the crane in Figure 1, considering the acceleration of the cart $\ddot{s}$ and the lengthening velocity $i$ as 
inputs of the system, is given by

$$
\dot{x}_{n l}=\left[\begin{array}{c}
\dot{s} \\
0 \\
0 \\
\dot{\theta} \\
-\frac{g \sin (\theta)}{l}
\end{array}\right]+\left[\begin{array}{c}
0 \\
1 \\
0 \\
0 \\
-\frac{\cos (\theta)}{l}
\end{array}\right] \ddot{s}+\left[\begin{array}{c}
0 \\
0 \\
1 \\
0 \\
-\frac{2 \dot{\theta}}{l}
\end{array}\right] \dot{l}
$$

where $x_{n l}=[s, \dot{s}, l, \theta, \dot{\theta}]^{T}$ is the state vector, and $g$ is the gravitational acceleration. The controlled variable of the system, that is the payload velocity, can be defined as $y_{n l}=\dot{s}+\dot{\theta} l \cos (\theta)+\dot{l} \sin (\theta)$.

In the case of constant cable length, that is when $i=0$, the model can be linearized with the assumption of small sway angles. With the vector of states for the linear model $x_{l}=[s, \dot{s}, \theta, \dot{\theta}]^{T}$, input the cart acceleration $\ddot{s}$ and controlled variable the payload velocity $y$, the system results in a state space description in the form

$$
\begin{aligned}
\dot{x}_{l} & =A x_{l}+B \ddot{s} \\
y_{l} & =C x_{l}
\end{aligned}
$$

with matrices

$$
A=\left[\begin{array}{cccc}
0 & 1 & 0 & 0 \\
0 & 0 & 0 & 0 \\
0 & 0 & 0 & 1 \\
0 & 0 & -\frac{g}{l} & 0
\end{array}\right], \quad B=\left[\begin{array}{c}
0 \\
1 \\
0 \\
-\frac{1}{l}
\end{array}\right], \quad C=\left[\begin{array}{l}
0 \\
1 \\
0 \\
l
\end{array}\right]^{T}
$$

\section{PROBLEM FORMULATION}

The position control of overhead cranes consists in point-to-point manoeuvres for which, knowing the initial and final position, the Optimal Control Problem (OCP) formulation is straightforward. With OIL cranes, the operator moves the load by means of forward and backward buttons on the remote controller, so that a position oriented approach is not possible. For these cases, a velocity control approach must be used.

The velocity reference of the payload depends on the command given by the operator, and can be defined as

$$
w:= \begin{cases}\alpha & \text { if forward button is pressed } \\ -\alpha & \text { if backward button is pressed } \\ 0 & \text { if no buttons are pressed }\end{cases}
$$

where $\alpha \in \mathbb{R}^{+}$is the desired absolute value of the velocity at steady state.

Similarly, the lengthening the cable is also controlled by the operator, so that the lengthening command can be defined as

$$
i:= \begin{cases}\beta & \text { if forward upward is pressed, } \\ -\beta & \text { if backward button is pressed, } \\ 0 & \text { if no buttons are pressed, }\end{cases}
$$

where $\beta \in \mathbb{R}^{+}$is the desired absolute value of the lengthening velocity at steady state.

\section{MPC FOR SIMULTANEOUS HOISTING AND TRAVELLING MANOEUVRES}

MPC consists in finding an optimal input sequence of $N_{c}$ future moves with respect to an OPC over a predictive horizons of $N_{p}$ time instants. Given the problem at hand, the OCP should minimize the velocity tracking error and the control effort. Moreover, in order to avoid big oscillations that could pose safety hazards, the difference between the position of the cart and the position of the payload is also weighted.

The cart acceleration input and the velocity of the cart have to be constrained in relation with the limits of the actuator (maximum cart acceleration and velocity). Therefore, the OCP can be formulated as

$$
\begin{array}{cll}
\underset{u}{\operatorname{minimize}} & \sum_{i=1}^{N_{p}}(y(k+i)-w(k))^{2}+ \\
+\lambda_{1} & \sum_{i=1}^{N_{p}}(\theta(k+i)-w(k))^{2}+\lambda_{u} \sum_{i=1}^{N_{c}}(\ddot{s}(k+i-1))^{2} \\
\text { subject to } & \dot{s}_{\min } \leq \dot{s}(k+i) \leq \dot{s}_{\max } & \forall i \in\left\{1, \ldots, N_{c}\right\} \\
& \ddot{s}_{\min } \leq \ddot{s}(k+i) \leq \ddot{s}_{\max } & \forall i \in\left\{1, \ldots, N_{c}\right\}
\end{array}
$$

where $\lambda_{1} \in \mathbb{R}^{+}$and $\lambda_{u} \in \mathbb{R}^{+}$are the weighting factors and $\dot{s}_{\text {min }}, \dot{s}_{\text {max }}$ and $\ddot{s}_{\text {min }}, \ddot{s}_{\text {max }}$ are the lower and upper bounds of the velocity and acceleration of the cart, respectively, while $y(k+1), \theta(k+1)$ and $\dot{s}(k+1)$ can be computed using models (1) and (2). 
TABLE I: Time instants for the ten simulations analysed.

\begin{tabular}{c|cccccc|c|c|c|c|c|c} 
& $\mathbf{1}$ & $\mathbf{2}$ & $\mathbf{3}$ & $\mathbf{4}$ & $\mathbf{5}$ & $\mathbf{6}$ & $\mathbf{7}$ & $\mathbf{8}$ & $\mathbf{9}$ & $\mathbf{1 0}$ \\
\hline$t_{1}$ & 1.83 & 2.20 & 1.50 & 1.92 & 4.37 & 1.77 & 1.90 & 1.68 & 1.91 & 2.74 \\
\hline$t_{2}$ & 4.21 & 3.60 & 3.75 & 2.24 & 2.28 & 1.72 & 2.53 & 2.54 & 3.77 & 3.67 \\
\hline$t_{3}$ & 12.24 & 14.69 & 14.65 & 11.73 & 14.61 & 14.91 & 12.75 & 11.44 & 12.03 & 12.63 \\
\hline$t_{4}$ & 12.57 & 11.51 & 13.62 & 12.13 & 11.40 & 13.75 & 13.50 & 12.20 & 12.48 & 12.34 \\
\hline
\end{tabular}

However, in the case of OIL control, the future velocity reference $w$ and length of the cable $l$ are not known in advance, as they depend on the operator decisions. For this reason, assumptions have to be made regarding the evolution of $w$ and $i$ along the predictive horizon.

As regards $w$, we resolve to the simple assumption that, at each control instant $k$, the velocity reference is constant fo the future $N_{p}$ sampling periods (i.e., $w(k+i)=w(k), \forall i=1 \ldots N_{p}$ ).

Regarding the length of the cable, the choice of how to consider the evolution of the cable length along the prediction horizon is arbitrary, but two main options seem to be reasonable:

Assumption A: to consider, at each control instant $k$, the length as constant and equal to $l(k)$ along the prediction horizon (i.e., $l(k+i)=l(k)$ and $\left.l(k+i)=0, \forall i=1 \ldots N_{p}\right)$,

Assumption B: to consider, at each control instant $k$, the lengthening velocity equal to $i(k)$ along the prediction horizon, starting from a cable length equal to $l(k)$ (i.e., $\left.l(k+i)=i(k), \forall i=1 \ldots N_{p}\right)$.

Given the aforementioned assumptions, different MPC strategies can be employed.

ADAPTIVE LINEAR MPC:

By assuming that the length of the cable is constant along the predictive horizon and equal to the current value $l(k)$, that is considering Assumption A, model (2) can be considered as a Linear Parameter Varying (LPV model) in $l$. The resulting controller is therefore an Adaptive MPC [20] (in which the predictive matrices are updated at every control instant with the current value of the cable length $l(k)$ ).

NONLINEAR MPC WITH NULL CABLE VELOCITY:

Similarly to the previous case, that is by making Assumption A, considering the length of the cable equal to $l(k)$ for the whole predictive horizon, also model (1) can be exploited, obtaining a Nonlinear MPC (NMPC) controller [21].

NONLINEAR MPC WITH CONSTANT CABLE VELOCITY: By making Assumption B, the use of model (1) allows to explicitly take into account the evolution of the cable over the predictive horizon. The resulting controller is again a NMPC one.

\section{RESUlts}

For comparison purpose, adaptive and nonlinear MPC are compared with the linear MPC in [18] by means of simulations considering different maneuvers with both horizontal travelling and hoisting. Nonlinear MPC approach has been simulated with both the Assumptions explained in Section III for the inclusion of the varying cable length along the prediction horizon.

For the simulations, a model of the overhead crane has been built using Simcenter Amesim ${ }^{\mathrm{TM}}$, which provides an easy way of specifying also parameters that are not present in the simplified models of Section II (e.g. masses, elasticity of the cable and frictions), allowing for more realistic simulations.

The mass of the cart has been set to $m_{C}=600 \mathrm{~kg}$, the mass of the payload to $m_{1}=100 \mathrm{~kg}$, the viscous friction of the cart and of the cable to $C_{c}=0.1 \mathrm{Ns} / \mathrm{m}$ and $C_{1}=0.01 \mathrm{Nms} / \mathrm{rad}$ respectively. The cable has been characterized with stiffness and viscous friction $K_{l}=1 \cdot 10^{6} \mathrm{~N} / \mathrm{m}$ and $\left.C_{l}=1000 \mathrm{Ns} / \mathrm{m}\right]$ per meter of cable. The Simcenter Amesim ${ }^{\mathrm{TM}}$ model of the crane is shown in Figure 2.

The control part has been developed using Simulink ${ }^{\mathrm{TM}}$, with a cycle time $T_{s}=20 \mathrm{~ms}$, chosen considering the slow dynamics of the system and the presence of already velocity and position control loops for the relatively fast dynamics of the cart. The weights of the MPC controller have been obtained through trial and error and have been set to $\lambda_{1}=1$ and $\lambda_{u}=0.2$, and control and prediction horizons $N_{c}=10$ and $N_{p}=100$ respectively. The Simulink controller and the Simcenter Amesim model run in cosimulation.

The manoeuvre that the crane has to follow can be divided into horizontal and vertical reference. At time $t_{1}$ the travelling button is pressed, setting the horizontal velocity reference to $\alpha=1 \mathrm{~m} / \mathrm{s}$. At time $t_{2}$, the payload is lifted at a constant velocity $\beta=1$ $\mathrm{m} / \mathrm{s}$ for 4 seconds. The travelling button is released at time $t_{3}$ and the lowering button is pressed at time $t_{4}$ and is released after 4 seconds. Ten different simulations have been analyzed; In order to take into account the operator unpredictability in the manoeuvre, the aforementioned time instants have been chosen in a random fashion, and their value are shown in Table I. For the sake of brevity, only Manoeuvre 1 is shown in the following plots, while the quantitative analysis is performed for all the manoeuvres in Table I.

The trajectories for Manoeuvre 1 are shown in Figure 3, and the time instants $t_{1, \ldots 4}$ are highlighted. In Figure 4 the tracking between reference and payload velocity is shown. 




Fig. 2: Simcenter Amesim model of the overhead crane

First, Figure 4 clearly shows that the linear MPC approach is not able to cope with varying cable lengths; when the cable

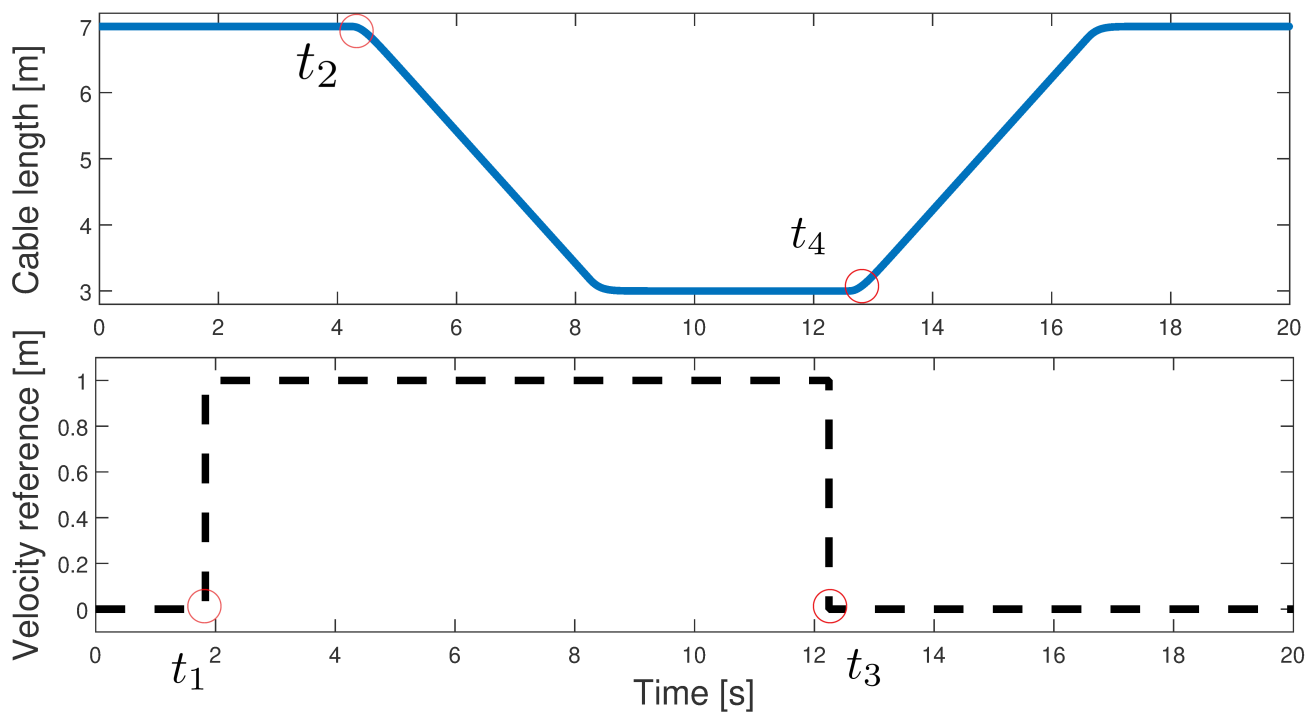

Fig. 3: Length of the cable and payload horizontal velocity reference over time.

is shorten, oscillations around the reference value are generated due to the difference between the overhead crane and the predictive model.

Second, it has to be noted that, when the length of the cable is considered constant along the prediction horizon and equal to the one measured at each control instant, adaptive and nonlinear MPC provide almost exactly the same performance. In fact, in this case, the only difference between the two approaches is the approximation for small sway angles introduced by the linearization. As the sway angles during industrial cranes manoeuvres are indeed small, the results do not differ significantly. On the other hand, when the length of the cable is considered to vary during the prediction horizon with the velocity measured at the present control instant, only NMPC can be exploited. Nonetheless, as the cable length does not vary significantly along the prediction horizon of 1 second $(\Delta l= \pm 1 \mathrm{~m})$, the effect of this variation does not influence the performance of the control in a significant way, so that the NMPC with constant cable velocity along the prediction horizon results to be comparable with the adaptive MPC. On the other hand, the adaptive MPC can rely on a simpler model of the crane, as the nonlinearities introduced by the varying length do not have to be addressed. 


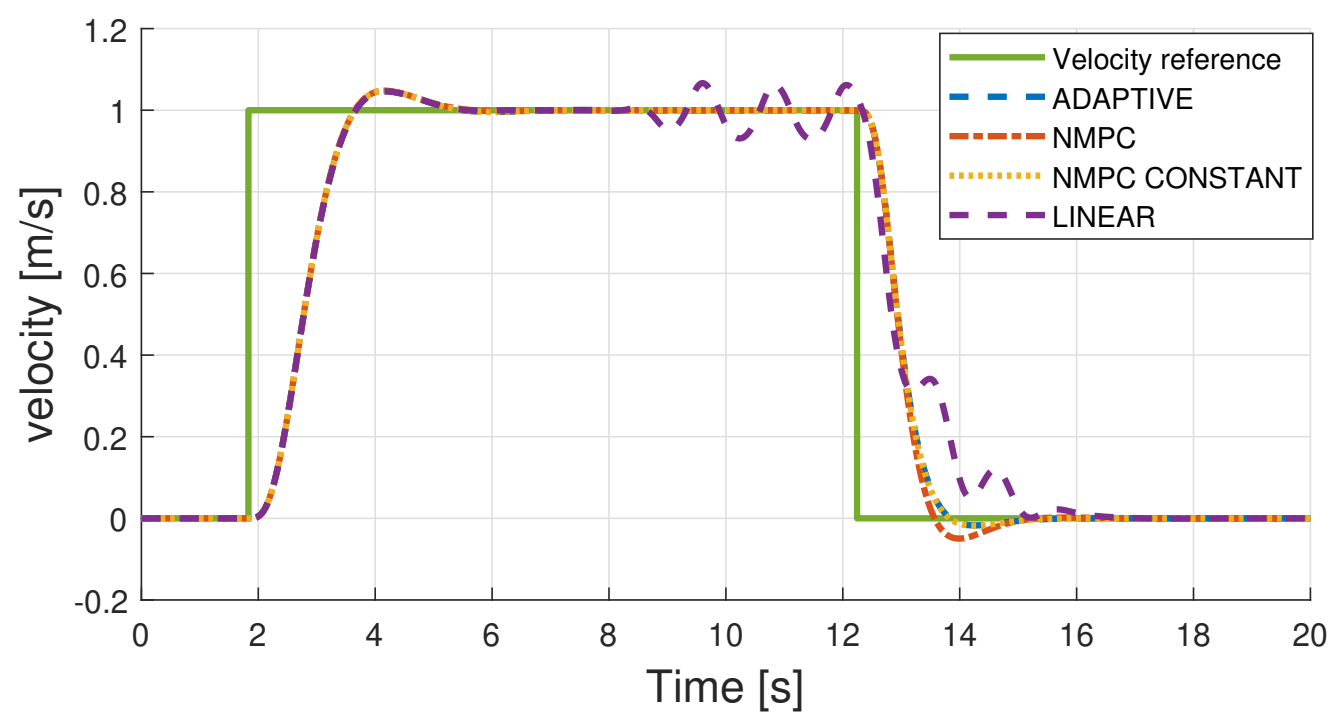

Fig. 4: velocity reference and payload velocity tracking performance with the simulated MPC approaches.

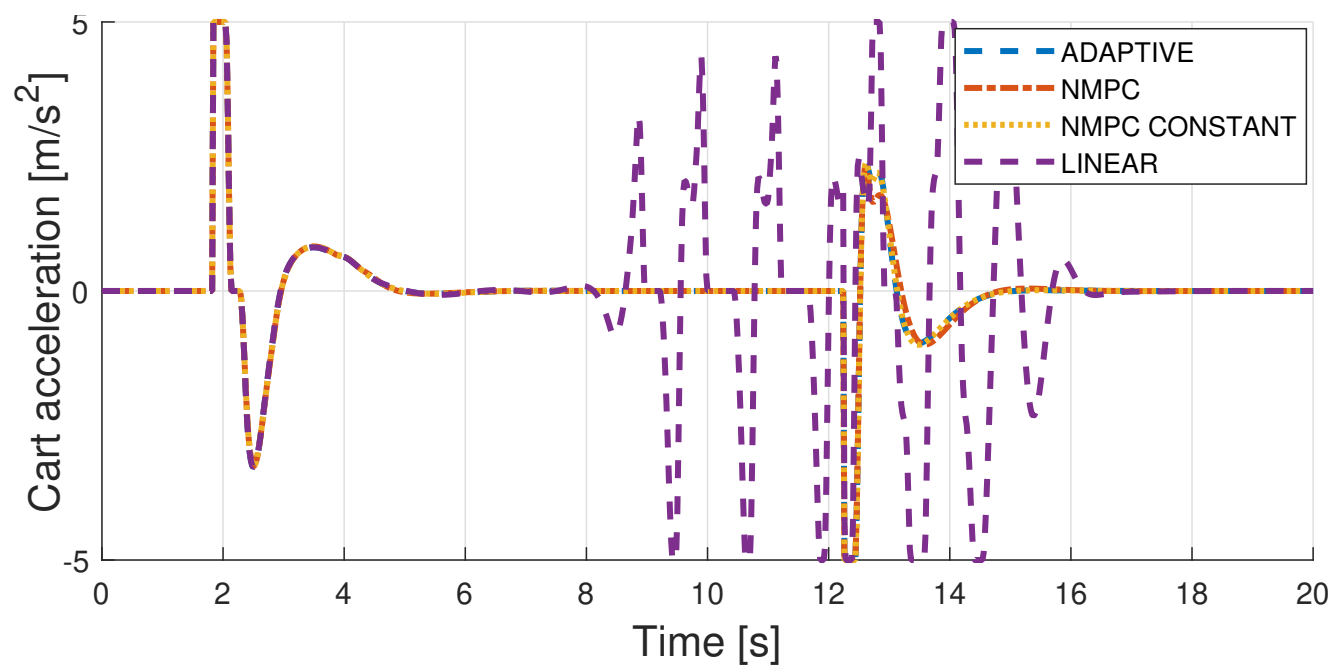

Fig. 5: Acceleration of the cart resulting with the simulated MPC approaches.

In Figure 5 the manipulated variable (acceleration of the cart) is shown for the simulated techniques. The constraint of $\ddot{s}_{\max }=5$ $\mathrm{m} / \mathrm{s}^{2}$ is always satisfied. In order to quantify the performance of the simulated techniques, two indices have been computed: the total Integral Absolute Error (IAE) and the position overshoot after the travelling button is released. Results show that the technique in [18] yields an unsatisfactory performance when dealing with simultaneous travelling and hoisting manoeuvres, as its IAE always exceeds the ones of the other techniques. Moreover, it is also not effective in containing the position drift of the payload after the operator releases the travelling button.

NMPC effectively reduces the IAE and the position drift with respect to the other two techniques which consider the cable length as invariant along the prediction horizon. Nonetheless, the performance of adaptive, NMPC and NPMC with constant cable length is very similar in all the ten simulations.

\section{CONSIDERATIONS ON PRACTICAL IMPLEMENTATION}

The results obtained through simulations allow us to make assessments on the practical implementation of the tested techniques on industrial setups. First of all, the increased complexity of the nonlinear model (1) is not justified by the slight increasing of control performance, as its velocity tracking performance is comparable with a linear adaptive approach. NMPC requires a computational effort which is hardly supported by off-the-shelf industrial crane controllers, while explicit formulations of linear MPC approaches are the standard for the control of various mechatronic systems.

Moreover, NPMC approaches require nonlinear quadratic solvers which, again, are rarely found on off-the-shelf industrial controllers. Even with an industrial hardware theoretically capable of supporting the increased computational effort required by a nonlinear approach, the implementation of nonlinear quadratic solver is non-trivial, requires a high-level programming 


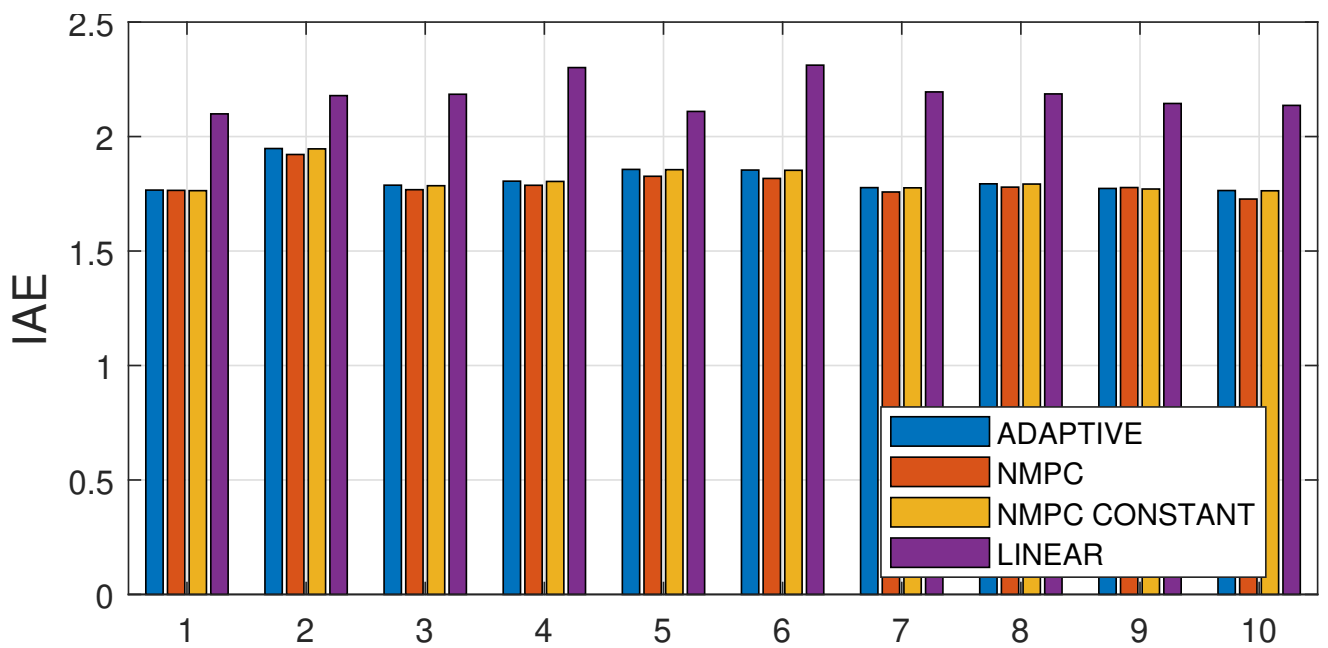

Fig. 6: Integral Absolute Error during the ten manoeuvres with the compared techniques.

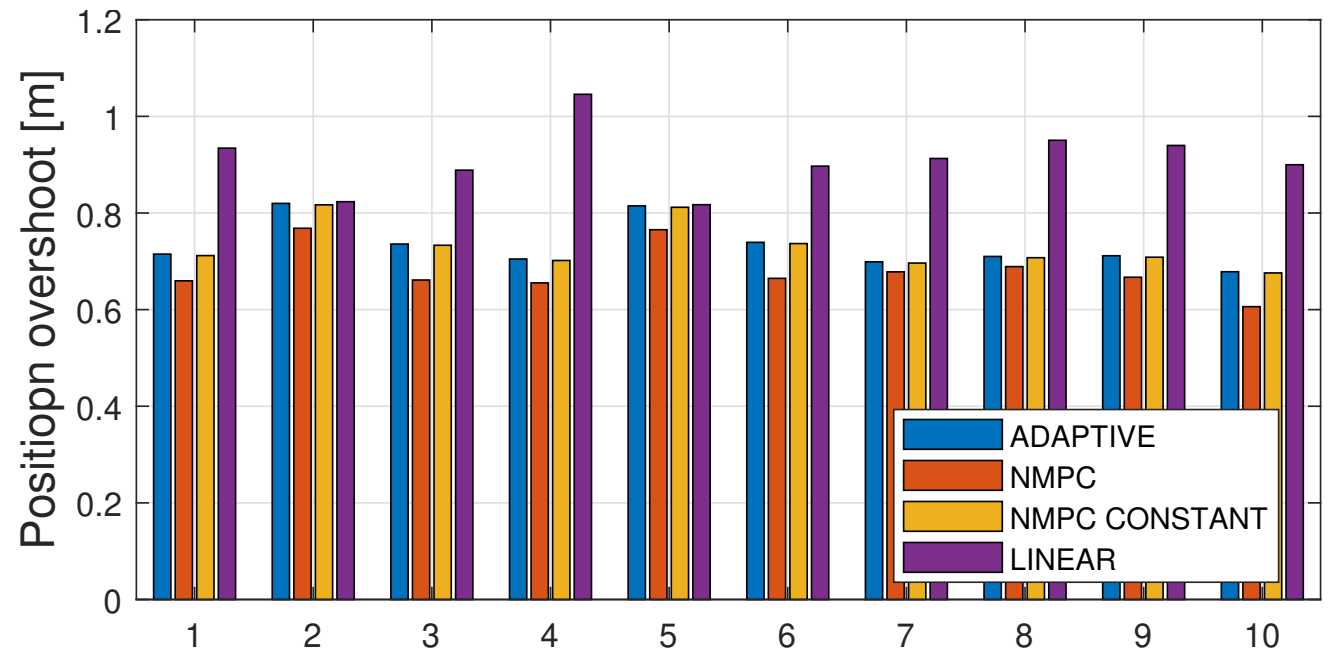

Fig. 7: Position overshoot after the release of the travelling button for the ten simulations analysed.

and the presence of usually proprietary and expensive external libraries.

On the other hand, while linear MPC can be easily implemented also in already in place control hardwares, Adaptive MPC theoretically requires the online calculation of the prediction matrices at each control instant, as they, again, depend on the actual length of the cable, leading to an heavy computational burden for the controller. Nonetheless, an easy workaround to this problem is to precompute a set of prediction matrices discretizing the length of the cable along its possible values and store them into the hardware memory [22]. It has to be noted that choosing the acceleration of the cart as input of the system represents a clear advantage for the implementation of the tested techniques in industrial cranes, as the underlying control structure of an off-the-shelf industrial gantry crane, based on cascade control for torque, velocity and (possibly) position control loops of the sliding cart, can be exploited. In fact, the cart acceleration computed by the MPC controller can be integrated in order to obtain velocity and position references for the already existing (and supposedly tuned) cascade control loops.

\section{CONCLUSIONS}

In this paper, a comparison of different MPC techniques (linear, adaptive and nonlinear MPC) applied to the control of operator maneuvered overhead cranes has been presented.

The obtained results show that a change in the length of the cable strongly affects the linear MPC approach proposed in [18], for which the performance strongly degrades when the cable length is varied. Adaptive and NMPC approaches can cope with cable length variations. Nonetheless, when the evolution of the cable length over the prediction horizon is not known in advance, such is the case of OIL overhead cranes, the use of NMPC does not introduce a significant advantage in performance with respect to an adaptive MPC approach. Considerations on a practical implementation on off-the-shelf industrial hardware of the tested techniques prove the adaptive MPC technique to be a better option than NMPC when controlling manually operated industrial overhead cranes. 


\section{REFERENCES}

[1] P. Hyla, "The crane control systems: A survey," in Prooceedings of the IEEE International Conference on Methods and Models in Automation and Robotics, 2012, pp. 505-509.

[2] W. Singhose, "Command shaping for flexible systems: A review of the first 50 years," International Journal of Precision Engineering and Manufacturing, vol. 10, no. 4, pp. 153-168, 2009.

[3] F. Padula, A. Visioli, D. Facchinetti, and A. Saleri, "A dynamic inversion approach for oscillation-free control of overhead cranes," in Proceedings of the IEEE International Conference on Emerging Technologies and Factory Automation, 2015, pp. 1-6.

[4] A. Piazzi and A. Visioli, "Optimal dynamic-inversion-based control of an overhead crane," IEE Proceedings-Control Theory and Applications, vol. 149, no. 5, pp. 405-411, 2002.

[5] M. Giacomelli, F. Padula, L. Simoni, and A. Visioli, "Simplified input-output inversion control of a double pendulum overhead crane for residual oscillations reduction," Mechatronics, vol. 56, pp. 37-47, 2018.

[6] H. M. Omar and A. Nayfeh, "Anti-swing control of gantry and tower cranes using fuzzy and time-delayed feedback with friction compensation," Shock and Vibration, vol. 12, no. 2, pp. 73-89, 2005.

[7] A. Marttinen, "Pole-placement control of a pilot gantry," in Proceedings of the American Control Conference, 1989, pp. 2824-2826.

[8] J. Smoczek and J. Szpytko, "Comparision of model predictive, input shaping and feedback control for a lab-scaled overhead crane," in Proceedings of the International Conference on Methods and Models in Automation and Robotics, 2016, pp. 288-293.

[9] L. Van den Broeck, M. Diehl, and J. Swevers, "Experimental validation of time optimal MPC on a flexible motion system," in Proceedings of the American Control Conference, 2011, pp. 4749-4754.

[10] J. Smoczek and J. Szpytko, "Soft-constrained predictive control for an overhead crane," Journal of KONES Powertrain and Transport, vol. 24, no. 3, 2017.

[11] D. Schindele and H. Aschemann, "Fast nonlinear MPC for an overhead travelling crane," 18th IFAC World Congress, vol. 44, no. 1, pp. 7963-7968, 2011.

[12] A. Khatamianfar and A. V. Savkin, "A new tracking control approach for 3d overhead crane systems using model predictive control," in Proceedings of the European Control Conference, 2014, pp. 796-801.

[13] Z. Wu, X. Xia, and B. Zhu, "Model predictive control for improving operational efficiency of overhead cranes," Nonlinear Dynamics, vol. 79, no. 4, pp. 2639-2657, 2015.

[14] Z. Wu and X. Xia, "Energy efficiency of overhead cranes," IFAC Proceedings Volumes, vol. 47, no. 3, pp. 19-24, 2014.

[15] D. Jolevski and O. Bego, "Model predictive control of gantry/bridge crane with anti-sway algorithm," Journal of Mechanical Science and Technology, vol. 29, no. 2, pp. 827-834, 2015.

[16] B. Käpernick and K. Graichen, "Model predictive control of an overhead crane using constraint substitution," in Proceedings of the American Control Conference, 2013, pp. 3973-3978.

[17] M. Vukov, W. Van Loock, B. Houska, H. J. Ferreau, J. Swevers, and M. Diehl, "Experimental validation of nonlinear MPC on an overhead crane using automatic code generation," in Proceedings of the American Control Conference, 2012, pp. 6264-6269.

[18] M. Giacomelli, M. Faroni, D. Gorni, A. Marini, L. Simoni, and A. Visioli, "Model predictive control for operator-in-the-loop overhead cranes," in Proceedings of the 23rd International Conference on Emerging Technologies and Factory Automation, 2018, pp. 589-596.

[19] W.-H. Chen, D. J. Ballance, and J. O'Reilly, "Model predictive control of nonlinear systems: Computational burden and stability," IEE Proceedings-Control Theory and Applications, vol. 147, no. 4, pp. 387-394, 2000.

[20] L. Chisci, P. Falugi, and G. Zappa, "Gain-scheduling MPC of nonlinear systems," International Journal of Robust and Nonlinear Control, vol. 13, no. 3-4, pp. 295-308, 2003.

[21] L. Magni, D. M. Raimondo, and F. Allgöwer, "Nonlinear Model Predictive Control," Lecture Notes in Control and Information Sciences, no. $384,2009$.

[22] J. Hu and B. Ding, "An off-line output feedback MPC strategy for nonlinear systems represented by quasi-LPV model," IFAC-PapersOnLine, vol. 51, no. 20, pp. 66-71, 2018. 